\title{
First observation of the depolarization of Thomson scattering radiation by a fusion plasma
}

\section{Giudigotti, L.}

2018

Giudigotti , L , JET Contributors \& Ahlgren , T 2018 , ' First observation of the depolarization of Thomson scattering radiation by a fusion plasma ', Nuclear Fusion , vol. 58 , no. 4 , 044003 . https://doi.org/10.1088/1741-4326/aab3fd

http://hdl.handle.net/10138/327067

https://doi.org/10.1088/1741-4326/aab3fd

unspecified

acceptedVersion

Downloaded from Helda, University of Helsinki institutional repository.

This is an electronic reprint of the original article.

This reprint may differ from the original in pagination and typographic detail.

Please cite the original version. 


\title{
First observation of the depolarization of Thomson scattering radiation by a fusion plasma
}

\author{
L. Giudicotti, ${ }^{1,2, *}$ M. Kempenaars, ${ }^{3}$ O. McCormack, ${ }^{2}$ J. Flanagan, ${ }^{3}$ \\ R. Pasqualotto ${ }^{2}$ and JET contributors ${ }^{\dagger}$
}

\author{
${ }^{I}$ Padova University, Department of Physics and Astronomy, Via Marzolo 8, 35131 Padova, Italy \\ ${ }^{2}$ Consorzio RFX, Corso Stati Uniti 4, 35127 Padova, Italy \\ ${ }^{3}$ CCFE, Culham Science Centre, Abingdon, Oxon, OX14 3DB, UK
}

\begin{abstract}
We report the first experimental observation of the depolarization of the Thomson scattering (TS) radiation, a relativistic effect expected to occur in very high $T_{e}$ plasmas and never observed so far in a fusion machine. A set of unused optical fibers in the collection optics of the HRTS (High Resolution Thomson scattering) system of JET has been used to detect the depolarized TS radiation during a JET campaign with $T_{e} \leq 8 \mathrm{keV}$. A linear polarizer with the axis perpendicular to the direction of the incident E-field was placed in front of a fiber optic pair observing a region close to the plasma core, while another fiber pair with no polariser simultaneously observed an adjacent plasma region. The measured intensity ratio was found to be consistent with the theory, taking into account sensitivity coefficients of the two measurement channels determined with post-experiment calibrations and Raman scattering. This depolarization effect is at the basis of polarimetric TS, an alternative method for the analysis of TS spectra that can provide significant advantages for $T_{e}$ measurements in very hot plasmas such as in ITER $\left(T_{e} \leq 40 \mathrm{keV}\right)$.
\end{abstract}

Keywords: Plasma diagnostics; Thomson scattering; Polarimetry; ITER.

Introduction - Incoherent Thomson scattering (TS) is a well established laser-aided diagnostic method for the measurement of electron temperature $\left(T_{e}\right)$ and electron density $\left(n_{e}\right)$ profiles in fusion plasmas. In all present day TS systems the measurement is based on the reconstruction of the doppler broadened frequency spectrum of laser radiation scattered by the plasma. Polarimetric TS is an alternative diagnostic technique in which the plasma $T_{e}$ and $n_{e}$ are obtained by measuring the depolarization of the TS radiation. This is a relativistic effect, first brought to attention by Theimer and Hicks [1] and later studied by other authors [2-6] who pointed out that the TS radiation from a very hot plasma is partially polarized and is constituted by the incoherent superposition of a completely and an incompletely (i.e. random) polarized components whose intensity ratio is largely independent from the radiation frequency and is a known function of the plasma $T_{e}$. This depolarization effect is weak in present day fusion experiments $\left(T_{e} \leq 10 \mathrm{keV}\right)$ and is in general not exploited, but it becomes significant in hotter plasmas where a measurement of the depolarization (the ratio between the intensity of the unpolarized component and the total intensity) of the TS radiation can provide a $T_{e}$ estimate 
independent from that obtained by the conventional spectral analysis [8].

Recently polarimetric TS has been proposed as a method suitable to overcome a limitation of the core plasma Thomson scattering (CPTS) system of the future ITER fusion experiment [7]. In CPTS the scattering angle $\theta$ is in the range $130^{\circ}-160^{\circ}$, and when $T_{e}$ is very high $\left(T_{e} \gtrsim 25 \mathrm{keV}\right)$ the width of the TS spectrum exceeds the detection spectral range of the measurement system which is restricted to the $500-1000 \mathrm{~nm}$ region by insufficient transmission of the collection and fiber optics and a high level of plasma light on the short wavelength side, and by the drop of the spectral quantum efficiency (QE) of the Si APD detectors on the long wavelength side. In these conditions a reliable reconstruction of the TS spectrum becomes difficult and the expected errors on the measured $T_{e}$ and $n_{e}$ obtained by the conventional spectral analysis increase significantly [8]. On the other hand, since the depolarization of the TS light is observed at any wavelength, polarimetric TS does not suffer from the limitation of the detection spectral range.

The best way to exploit the depolarization effect in CPTS is to implement the hybrid TS technique suggested in Refs. [9-11]. In this scheme an additional detection channel, specially designed to measure the unpolarized component of the TS radiation (the polarimetric channel), is added to the conventional set of spectral channels of a typical TS filter polychromator. This set-up provides an additional signal, increasing with $T_{e}$, that may significantly improve the accuracy of $T_{e}$ and $n_{e}$ measurements starting from $T_{e} \simeq 25 \mathrm{keV}$. The expected improvement of the $T_{e}$ fractional error is $\sim 1.5$ at $40 \mathrm{keV}$ for an input laser beam linearly polarized in the direction perpendicular to the scattering plane (the typical TS conditions) and is even higher (up to a 2.2 factor at $40 \mathrm{keV}$ ) if the input laser beam has a circular polarization [11].

In spite of its interest for diagnostic applications, so far this depolarization effect has never been experimentally observed in a fusion plasma. In this letter we report the first quantitative experimental observation of the depolarization of TS radiation, in agreement with the theory, obtained in the High Resolution Thomson Scattering (HRTS) diagnostic of the JET fusion experiment.

The experiment set-up - The HRTS diagnostic on the JET experiment is a conventional $\theta \sim 90^{\circ}$ incoherent TS system [12]. It uses a $5 \mathrm{~J}, 20 \mathrm{~Hz} \mathrm{Nd}$ :YAG $\left(\lambda_{0}=1064 \mathrm{~nm}\right)$ to measure $T_{e}$ and $n_{e}$ profiles in up to 63 spatial positions along the outer radius of the plasma $(R=2.9-3.9 \mathrm{~m}$ ), with a resolution of $\sim 15 \mathrm{~mm}$. Light from these scattering volumes is collected through a vertical main upper port and imaged onto a linear array of $1 \mathrm{~mm}$ diameter fibers, located on the torus hall wall, with two fibers used for each scattering volume. Three fiber pairs of different length are combined in input to each of the 21 filter polychromators so that the TS signals from three different scattering volumes arrive separated in time and can be measured by the same polychromator. Transient digitisers record the signal at $1 \mathrm{Gs} / \mathrm{s}$, which provides resolution between the three combined, time-delayed signals.

Figure 1 shows the cross section of the plasma, the laser path and the position of the scattering volumes used for our experiment. Two contiguous scattering volumes were chosen, close to the plasma core where the highest temperatures are expected. Fibers F124 and F125 $(R=2.95 \mathrm{~m})$ were both fitted with a linear polarizer in front of them, oriented with the axis perpendicular to the linear polarization of the completely polarized 
component of the TS light (polarimetric channel). Fibers F127 and F128 with no polarizer, were used to observe a control scattering volume $(R=2.93 \mathrm{~m})$ close enough to the previous one to assume that in stable plasma conditions both have the same $T_{e}$ and $n_{e}$. The two fibers pairs were connected to the same polychromator, with an extra length of $60 \mathrm{~m}$ for the control fibers, providing a $150 \mathrm{~ns}$ time delay, so that the two signals can be measured at different times by the same detector.

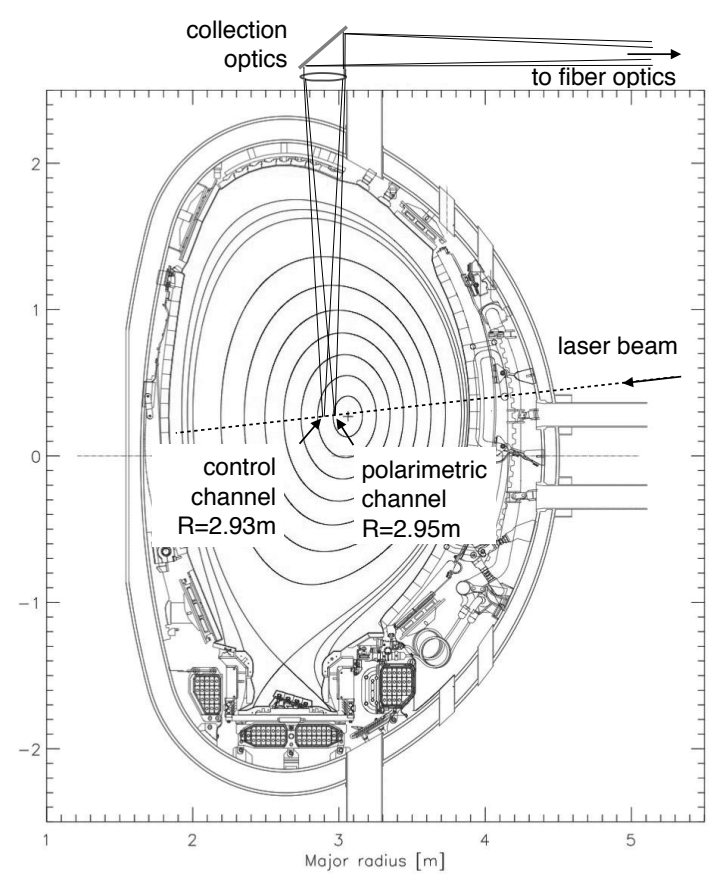

FIG. 1 - Cross section of the JET vacuum vessel, showing the laser path in the plasma and the position of the scattering volumes of the two measurement channels.

A standard HRTS polychromator samples the TS spectrum in four wavelength channels [12]. For our measurements, we used only the first channel, replacing its bandpass filter by a notch filter that just removes the stray light rejecting a very narrow region around the Nd:YAG laser line at $1064 \mathrm{~nm}$. In this way channel 1 measures the wavelength integrated intensity of both signals.

An important feature of this set-up is that the polarimetric and control signals share the same collection optics (window lens and mirror), are carried by fiber optics having a transmission with the same spectral dependence and are measured at different times by the same filtered detector. Therefore any dependence from the polychromator parameters, including the detector $\mathrm{QE}$, and from the spectral transmission of the entire detection system, cancels out in their ratio and to a very good approximation can be neglected.

A simple model of depolarization measurements - Let's define $I_{\perp}$ and $I_{0}$ as the TS signals detected by the polarimetric and by the control channel respectively and let $N_{\perp}$ and $N_{\|}$be the number of photons scattered by the plasma in the parallel and perpendicular polarization states. Due to the non-ideal behaviour of the polarizer and to small additional depolarization effects in the collection optics, the signal $I_{\perp}$ will collect also a small fraction $k$ of the photons emitted in the parallel polarization. Let's call $k$ the system depolarization factor. The $I_{0}$ signal, receiving both polarizations, will comprise of $N_{\|}$and $N_{\perp}$. Taking into account that 
the two channels can have different sensitivities $C_{\perp}$ and $C_{0}$ the measured signals and their ratio can be written as:

$$
\begin{gathered}
I_{\perp}=C_{\perp}\left(N_{\perp}+k N_{\|}\right), \quad I_{0}=C_{0}\left(N_{\perp}+N_{\|}\right) \\
\frac{I_{\perp}}{I_{0}}=C \frac{N_{\perp}}{N_{\perp}+N_{\|}}+C k \frac{N_{\|}}{N_{\perp}+N_{\|}}
\end{gathered}
$$

in which $C=C_{\perp} / C_{0}$ is the ratio between the channel sensitivities. Given that $N_{\perp} \ll N_{\|}$we can assume $N_{\|} /\left(N_{\perp}+N_{\|}\right) \simeq 1$ to a very good approximation. We also define the depolarization ratio $R\left(T_{e}\right)=N_{\perp} /\left(N_{\perp}+N_{\|}\right)$which is a function of $T_{e}$ only, so that Eq. (2) can be written as:

$$
\frac{I_{\perp}}{I_{0}}=C R\left(T_{e}\right)+C k
$$

The photon signals $N_{\perp}$ and $N_{\|}$are calculated by using the Stokes vector $S\left(T_{e}\right)$ of the TS radiation which is given by:

$$
S\left(T_{e}\right)=M\left(\theta, T_{e}\right) \cdot S_{I N}
$$

Here $M\left(\theta, T_{e}\right)$ is the frequency integrated TS Mueller matrix [5,6] and $S_{I N}=(1,1,0,0)^{\mathrm{T}}$ is a unit Stokes vector representing the incident laser radiation linearly polarized in the direction perpendicular to the scattering plane. Given the structure of the $M\left(\theta, T_{e}\right)$ matrix, the two first components of the Stokes vector are:

$$
S_{0}=M_{00}+M_{01}, \quad S_{1}=M_{01}+M_{11}
$$

and the other two are null. The photon signals can then be written [13]:

$$
N_{\perp}=\frac{1}{2}\left(S_{0}-S_{1}\right), \quad N_{\|}=\frac{1}{2}\left(S_{0}+S_{1}\right)
$$

and the depolarization ratio is calculated as

$$
R\left(T_{e}\right)=\frac{M_{00}-M_{11}}{2\left(M_{00}+M_{01}\right)}
$$

The data analysis can be greatly simplified by introducing a linear approximation for $R\left(T_{e}\right)$. In fact from a simple best-fit of $R\left(T_{e}\right)$ data we find that in the $1-8 \mathrm{keV}$ range $R\left(T_{e}\right) \simeq \alpha T_{e}$ with $\alpha=1.893 \times 10^{-6} \mathrm{eV}^{-1}$ and a maximum deviation of $2.5 \%$ (at $8 \mathrm{keV}$ ) between the approximate and the true values. Then Eq. (7) can be written

$$
\frac{I_{\perp}}{I_{0}}=C \alpha T_{e}+C k
$$


Therefore the ratio between the signals measured by the polarimetric and the control channels has a simple linear dependence on $T_{e}$, in which the slope and the intercept are determined by the channel sensitivity ratio $C$ and the system depolarization factor $k$ respectively.

Data analysis - The depolarization measurements were taken during the C36B Deuterium JET campaign, where temperatures up to $8 \mathrm{keV}$ were recorded. This campaign produced a data set of $\sim 300$ useable plasma pulses. Within each plasma pulse a time window was selected to extract data only during a flat top of $T_{e}$ of 1-10 s duration, depending on each pulse. During each time window the TS laser was fired several times and the data acquisition system was optically triggered by the laser itself to acquire the combined signals from the two channels in a $500 \mathrm{~ns}$ time window around each pulse pair. The single polarimetric signal was often buried in the plasma light noise, but both pulses were very efficiently recovered by summing up in sync many pulse pairs and fitting the cumulated waveform with a pair of gaussian pulses (Figure 2). Before each plasma pulse the TS laser was fired several times in vacuum, to record the signal due to the residual stray light which is later subtracted from the main signal. Data were normalized to the energy of each laser pulse.

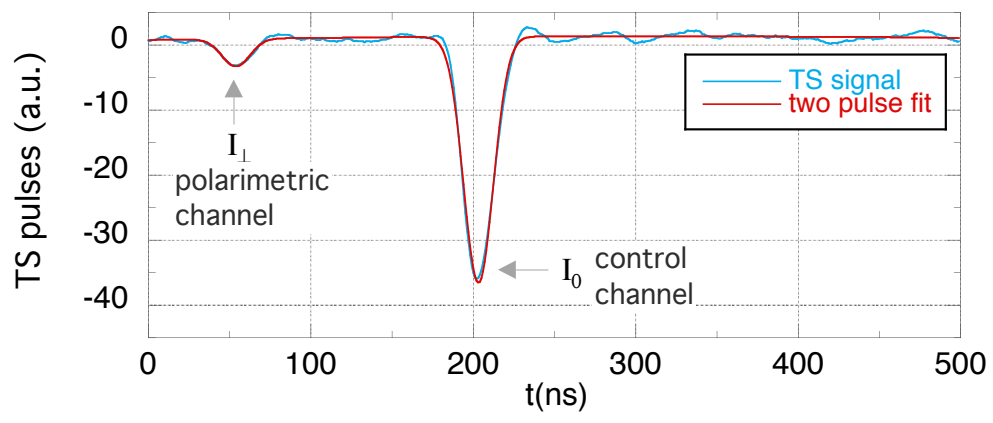

FIG. 2 - Typical pulse signal averaged over a flat top time window after stray light subtraction. The small peak is the signal from the polarimetric channel $I_{\perp}$, the large peak is the signal from the control channel $I_{0}$.

The four fibers cover a radial range of just over $3 \mathrm{~cm}$ near the plasma core around the radial position at $R=2.941 \mathrm{~m}$. For this plasma region we assumed a single $T_{e}$ value corresponding to the $T_{e}$ measured by the HRTS system in the same time window in the closest radial position which is centered at $R=2.975 \mathrm{~m}$. Figure 3 shows a plot of the measured values of the signal ratio $I_{\perp} / I_{0}$ as a function of $T_{e}$. According to Eq. (8) data were fitted with a simple linear function $f\left(T_{e}\right)=a+b T_{e}$ finding $a=(4.69 \pm 0.65) \times 10^{-2}$ and $b=(1.02 \pm 0.14) \times 10^{-5} \mathrm{eV}^{-1}$. These gave $C=5.45 \pm 0.77$ and $k=(8.61 \pm 2.37) \times 10^{-3}$. 


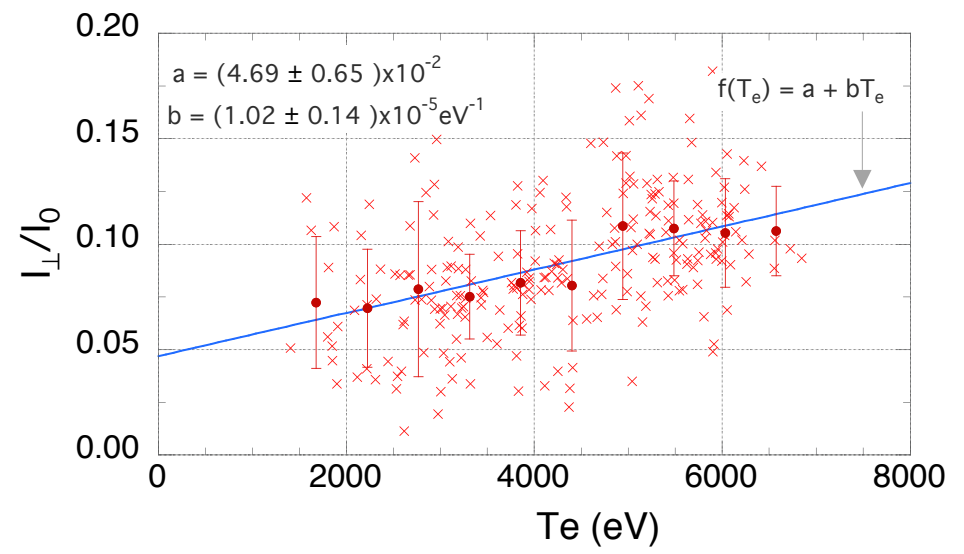

FIG. 3 - The measured signal ratio as a function of the plasma $T_{e}$. The $a$ and $b$ parameters of the fitted straight line are determined by a simple, unweighted linear fit of all the data points (crosses). The dots with error bars represent the average values and the standard deviation of the data contained in 10 equally spaced bins on the $T_{e}$ axis. The binned data are plotted only to highlight the linear behaviour and are not used for the best-fit.

In spite of the considerable dispersion of the single points around the fitted function, the linear dependence expected from Eq. (8) is confirmed. This is more evident considering the points representing the binned data. However in order to verify that the observed linear behaviour is really due to the depolarization of the TS radiation, it is necessary to ascertain that the values of $C$ and $k$ determined by the fit are consistent with the parameters of the experimental set-up.

Post-experiment and Raman calibrations - The measured value of $C$ indicated that the sensitivity of the polarimetric channel is more than 5 times higher than that of the control channel. At first this seemed somewhat surprising considering that both signals were measured at different times in the same polychromator, with the same filtered detector. However it was soon realized that such a difference in sensitivity may be due to a difference in the imaging efficiency of the scattered volumes into the fiber optics. Therefore a careful calibration of the fiber collection efficiency has been performed at the end of the experimental campaign. For this we used a broadband light source, constituted by a large integrating sphere with a $100 \mathrm{~W}$ tungsten light bulb, located inside the JET vacuum vessel and shining unpolarized light upwards into the TS collection optics system. The output from each TS fiber was individually measured as a function of wavelength by a monochromator (Figure 4).

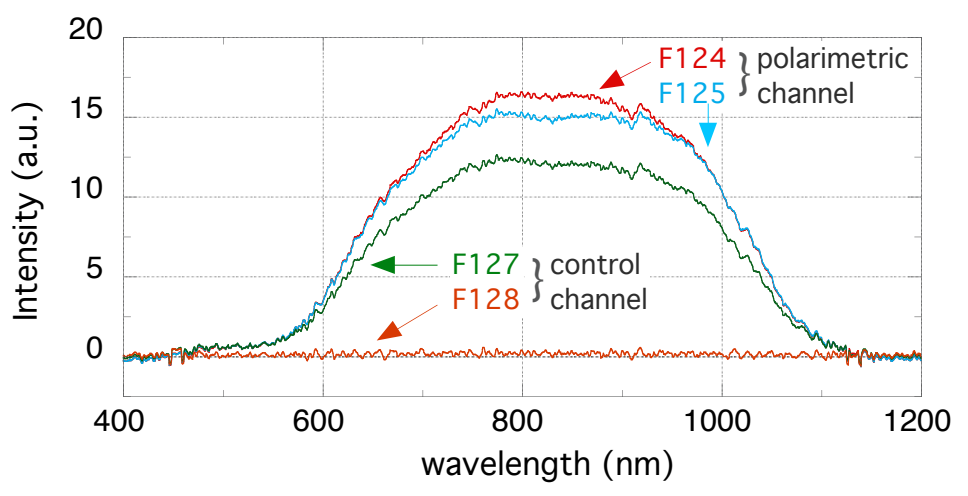

FIG. 4 - Signal from the calibration light source measured as a function of the wavelength at the end of the four fibers (polychromator input) of our experiment. Fibers F127 and F128 carry the signal to the control channel and fibers F124 and F125 carry the signal to the polarimetric channel. 
Figure 4 clearly shows that F128, one of the two fibers carrying the signal to the control channel, is virtually blind, and also the signal from F127, that should be twice as high as that of F124 or F125, is remarkably attenuated. A subsequent check of the fiber alignment, made sending light back through the fibers, from the polychromator to the vacuum vessel, confirmed a misalignment, partial for F127 and almost complete for F128, with the collection window. This explains the remarkable difference between the sensitivity of the two measurement channels. Eventually it was realized that this reduced sensitivity of the control channel was serendipitously useful to avoid the saturation of the data acquisition system by the control signal while conserving a good sensitivity in the polarimetric channel.

To calculate the sensitivity ratio from the signals of the calibration source, we have to consider that in our TS measurements the detected spectrum is contained within the region $600 \mathrm{~nm}<\lambda<1050 \mathrm{~nm}$. Therefore the quantities of interest are the signals carried by the fiber pair associated to each measurement channel integrated over this wavelength range: $I_{\perp}=\int_{600}^{1050}\left(F_{124}+F_{125}\right) d \lambda$ and $I_{0}=\int_{600}^{1050}\left(F_{127}+F_{128}\right) d \lambda$. For the calibrating source $N_{\perp}=N_{\|}$and the system depolarization factor $k$ can be neglected. The measured intensities are related to the photon fluxes emitted by the calibrating source by $I_{\perp}=C_{\perp} N_{\perp}$ and $I_{0}=C_{0}\left(N_{\perp}+N_{\|}\right)$. For the calibrating source $N_{\perp} /\left(N_{\perp}+N_{\|}\right)=\frac{1}{2}$ and we find the sensitivity ratio $C=2 \int_{600}^{1050}\left(F_{124}+F_{125}\right) d \lambda / \int_{600}^{1050}\left(F_{127}+F_{128}\right) d \lambda=5.28 \pm 2 \%$, with the error estimated by the noise of the fiber signals. This value is consistent with that determined by the best-fit of Figure 3 .

Another independent measurement of the channel sensitivity ratio has been obtained from Raman scattering. This is a typical calibration technique of TS systems in which the calibrating light is obtained by Raman scattering from a molecular gas $\left(\mathrm{N}_{2}\right.$ in case of JET) filling the vacuum vessel. Raman calibration is used to check the alignment of the collection optics and to absolutely calibrate the $n_{\mathrm{e}}$ measured by TS [14,15]. A retrospective examination of Raman scattering data recorded before the TS campaign showed that only four Raman data sets were available for a calibration of the sensitivity ratio of depolarization measurements. An example of a Raman signal pair is shown in Figure 5.

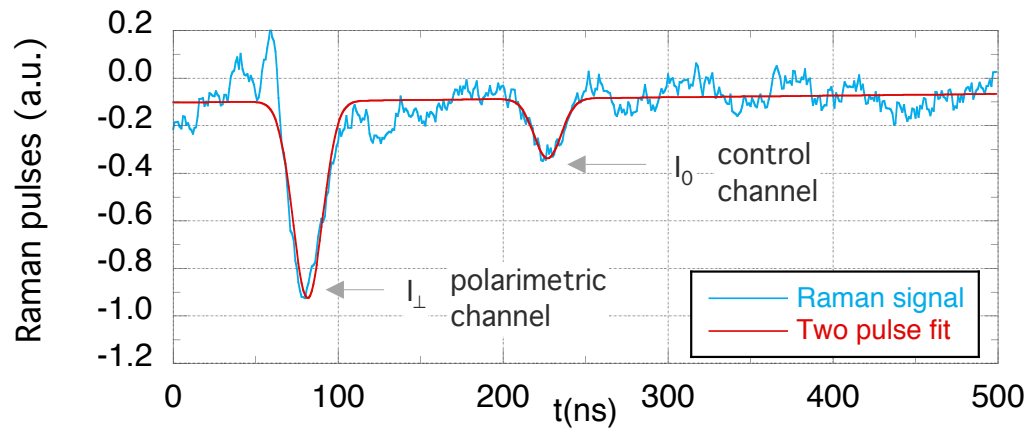

FIG. 5 - Example of a pulse pair from Raman scattering measurements. The difference in the sensitivity of the two channels is evident from the fact that the signal of the polarimetric channel (that collects only the light with perpendicular polarization) appears considerably higher than that of the control channel that collects the total intensity.

The average measured value of the Raman signal ratio is $I_{\perp} / I_{0}=2.88 \pm 0.78$. The number of photons scattered in the two channels has to be calculated as in Eqs. (4) through (7), but using the Mueller matrix of 
Raman scattering [16,17]. We find the Raman depolarization ratio $N_{\perp} /\left(N_{\perp}+N_{\|}\right)=3 / 7$ from which the channel sensitivity ratio from Raman measurements is $C=\frac{7}{3}\left(I_{\perp} / I_{0}\right)=6.73 \pm 1.83$. In spite of the larger error, this value is also consistent with the value determined by the broadband calibration source and with the channel sensitivity ratio determined by the best-fit of Figure 3 .

With regards to the system depolarization factor $k$, we first measured the contrast ratio of the linear polarizer used in the polarimetric channel. This was determined experimentally in the lab, by measuring the light from the tungsten lamp leaking from a pair of crossed polarizers equal to that used in the polarimetric channel as a function of the wavelength. The average value of the contrast ratio in the wavelength region $600-1050 \mathrm{~nm}$ was found to be $1.6 \times 10^{-3} \pm 10 \%$. This is consistent with the value of the contrast ratio in the polarizer data sheets [18] but is a factor 5 lower than the value of $k$ determined by the best-fit of Figure 3. However the polarizer contrast ratio only sets a lower limit to the system depolarization factor, which is supposedly affected also by the additional depolarization introduced by the optical elements (the vacuum window, lens and two mirrors) of the collection optics system. So far it has not been possible to measure this contribution, but the difference between the measured contrast ratio of the polarizer and the value of $k$ determined from the best-fit of Figure 3 may well be accounted for by this effect.

Conclusions - The results of post-experiment calibrations and Raman scattering are consistent with the values of the channel sensitivity ratio $C$ and of the system depolarization factor $k$ determined by the best-fit of polarimetric data. This confirms that we have actually measured the depolarized TS radiation and, as far as we know, this is the first time this is quantitatively observed in a magnetic fusion plasma and found in agreement with the theory. Our experiment also showed that by cumulating many laser pulses in sync, the polarimetric signal can be detected also for $T_{e}<10 \mathrm{keV}$, when it is buried in the plasma light noise.

These results confirm that polarimetric TS is a useful technique that can supplement the conventional spectral analysis or even provide an independent $T_{e}$ measurement in very hot fusion plasmas such as those of ITER. Finally we point out that the set-up of our JET experiment was not optimized for the detection of the unpolarized TS radiation. In fact, for $\theta \simeq 90^{\circ}$ and $T_{e}=8 \mathrm{keV}$ the intensity of the unpolarized component of TS radiation can be increased by $\sim 3$ times by using an input laser beam linearly polarized in a direction forming an angle $\psi=87^{\circ}$ with the perpendicular to the scattering plane. In our JET experiment this was not possible because it would have considerably reduced the accuracy of the conventional (spectral) $T_{e}$ profile measurements during the JET campaign.

Acknowledgments - The authors are indebted to M. Valisa who gave useful suggestions for improving the manuscript. This work has been carried out within the framework of the EUROfusion Consortium and has received funding from the Euratom research and training programme 2014-2018 under grant agreement No 633053. The views and opinions expressed herein do not necessarily reflect those of the European Commission. 
* Corresponding author leonardo.giudicotti@unipd.it

† See the author list of "X. Litaudon et al. 2017 Nucl. Fusion 57, 102001"

[1] O. Theimer and W. Hicks, Depolarization of light scattered by a relativistic plasma, Phys. Fluids 11, 1045 (1968) http://dx.doi.org/10.1063/1.1692039.

[2] F. Orsitto and N. Tartoni, Proposal for a new electron temperature diagnostic for fusion reactors, Rev. Sci. Instrum. 70, 798 (1999) http://dx.doi.org/10.1063/1.1149414.

[3] S. E. Segre and V. Zanza, Polarization of radiation in incoherent Thomson scattering by high temperature plasma, Phys. Plasmas 7, 2677 (2000) http://dx.doi.org/10.1063/1.874110

[4] E. Parke, V. V. Mirnov and D. J. Den Hartog, A polarization-based Thomson scattering technique for burning plasmas, JINST 9 C02030 (2014). https://doi.org/10.1088/1748-0221/9/02/C02030

[5] V. V. Mirnov, D. J. Den Hartog, and E. Parke, Exact relativistic expressions for polarization of incoherent Thomson scattering, Phys. Plasmas 23, 052108 (2016) http://dx.doi.org/10.1063/1.4948488.

[6] V. V. Mirnov and D. J. Den Hartog, Polarization of incoherent Thomson scattering for electron temperature measurement, Plasma Phys. Control. Fusion 59, 063001 (2017) https://doi.org/10.1088/1361-6587/aa61e2.

[7] M. Bassan, P. Andrew, G. Kurskiev, E. Mukhin, T. Hatae, G. Vayakis, E. Yatsuka and M. Walsh, Thomson scattering diagnostic systems in ITER, JINST 11, C01052 (2016) https://doi.org/10.1088/1748-0221/11/01/C01052.

[8] L. Giudicotti, M. Bassan, F. P. Orsitto, R. Pasqualotto, K. Kempenaars and J. Flanagan, Conceptual design of a polarimetric Thomson scattering diagnostic in ITER, JINST 11, C01071 (2016) https://doi.org/10.1088/1748-0221/11/01/C01071.

[9] L. Giudicotti, R. Pasqualotto and O. McCormack, Extending the $T_{e}$ range of the ITER core Thomson scattering system by detecting the unpolarized scattering radiation, $21^{\text {st }}$ Topical Conf. on High-Temperature Plasma Diagnostics - HTPD 2016 Madison, Poster 6.2.38 (2016).

[10] G. S. Kurskiev et al., ITER core Thomson scattering: objective and error analysis, $26^{\text {th }}$ IAEA Fusion Energy Conference (FEC 2016) FIP/P4-272 (2016).

[11] L. Giudicotti, Depolarization measurements in ITER Core Plasma TS, ITER report IDM-UD7YKR (2017).

[12] R. Pasqualotto et al., High resolution Thomson scattering for Joint European Torus (JET), Rev. Sci. Instrum. 75, 3891 (2004) http://dx.doi.org/10.1063/1.1787922.

[13] D. Goldstein, Polarized light, Marcel Dekker Inc., New York (2003).

[14] F. Flora and L. Giudicotti, Complete calibration of a Thomson scattering spectrometer system by rotational Raman scattering in $\mathrm{H}_{2}$, Appl. Opt. 26, 4001 (1987) https://doi.org/10.1364/AO.26.004001.

[15] R. Scannell, M. Beurskens, M. Kempenaars, G. Naylor, M. Walsh, T. O’Gorman, and R. Pasqualotto, Absolute calibration of LIDAR Thomson scattering systems by rotational Raman scattering, Rev. Sci. Instrum. 81, 045107 (2010) http://dx.doi.org/10.1063/1.3374111.

[16] L. Giudicotti and R. Pasqualotto, Rotational Raman scattering as a source of polarized radiation for the calibration of polarization-based Thomson scattering, Plasma Phys. Control. Fusion 57, 035001 (2015) 
https://doi.org/10.1088/0741-3335/57/3/035001.

[17] L. Giudicotti and R. Pasqualotto, On the calibration of polarimetric Thomson scattering by Raman polarimetry, Plasma Phys. Control. Fusion 57, $125015 \quad$ (2015) https://doi.org/10.1088/0741-3335/57/12/125015.

[18] Model PPL05A from Moxtek Inc, Orem, UT, USA, www.moxtek.com. 